\title{
Intravesical Cacillus Calmette-Guerin Therapy Failure
}

National Cancer Institute

\section{Source}

National Cancer Institute. Intravesical Cacillus Calmette-Guerin Therapy Failure. NCI

Thesaurus. Code C160492.

Failure to achieve a disease-free state following BCG treatment. 